\title{
Zwei Bemerkungen zu Gebel es-Silsila Nr. 100
}

The stela of Shoshenq I at Gebel es-Silsila contains a verb $m t j$ that has been considered as an early example of $m t j / m t r$, 'to be pleased' (attested mainly in demotic). This paper tries to show that it belongs to the stem $m t n / m t n$, 'to reward'. A writing of the infinitive of $j r j$ on this same stela as $j r . n n$ is attested several times at the beginning of the Twenty-second Dynasty.

\section{Ein verkannter Beleg des Verbs $m t n / m t n$, 'belohnen'}

IN der zuletzt von R. A. Caminos publizierten Felsstele Schoschenks I. vom Gebel es-Silsila ${ }^{5}$ erscheinen in zwei parallel gebauten Passagen in Z.35 und Z.38 folgende Sätze: 'Mögest du [=Amun] veranlassen, daß die in Millionen Jahren kommenden (Generationen) sagen werden: "Nützlich ist es, Amun zu dienen", 光 (Z.38). Caminos übersetzt diese Sätze: 'Mayest thou be pleased to accord me a great reign' bzw. 'might and victory' (p. 50) ${ }^{6}$ und erläutert (p. 54) 'lit. "mayest thou agree upon making for me a great reign". The meaning of $m t y$ here and below line $\left(3^{8}\right)$ is hesitatingly suggested by Sir Alan Gardiner in the light of demotic $m t i$ "to be pleased"...Preposition $n$ is for $m$ introducing the object of ir $(t)^{\prime}$.

${ }^{1}$ Cf. PM rv, 46; J. Málek, LÄ v, 355-7, n. I8; J. Baines and J. Málek, Atlas of Ancient Egypt (Oxford, I980), I70.

${ }^{2}$ Notably the statuette of Osorkon III from the Karnak cachette (CG 42197: PM II ${ }^{2}$, I43) which, as Cyril Aldred has noted (Egyptian Art (London, I980), 210; cf. Les Pharaons, III. L’Égypte du crépuscule (Paris, I980), I28), 'Even in such details as the design of the belt, the uraeus and ear-lobes, ... follows a mid-Eighteenth Dynasty prototype.' C'f. also J. R. Harris, Egyptian Art (London, I966), 4I, pl. 50.

${ }^{3}$ Cf. H. W. Müller, BiOr io (1955), 32; B. V. Bothmer, ${ }^{2} E A_{4} 6$ (ig6o), $3 \mathrm{ff}$.

${ }^{+}$Cairo CG 42228, the seated statue of Shebensopdet, daughter of Nimlot, son of Osorkon III, from the Karnak cachette (PM I², I49; Ramadan el Sayed, ASAE 65 (1983), III ff.; K. Jansen-Winkeln, Die ägyptische Biographien der 22. und 23. Dynastie (Wiesbaden, 1985), 11, $520 \mathrm{ff}$., pls. $37-40$ ).

5 yEA 38 (1952), 46-61.

${ }^{6}$ Entsprechend auch U. Kaplony-Heckel in: Texte aus der Umwelt des Alten Testaments, I (Gütersloh, I985), $555^{-6 .}$ 
In der hier postulierten Bedeutung 'einverstanden, zufrieden sein' kommt das sonst in der klassischen Sprachstufe durch 'richtig sein, genau sein' wiedergegebene $m t{ }^{1}$ tatsächlich schon in der 22. Dynastie vor, s. I. E. S. Edwards, Oracular Amuletic Decrees of the Late New Kingdom (London, I960), I, 48-9, n. 25=II, pl. xvii: jw.j $(r)$ djt mtj ḩstj NN ... (m) jm.w (L7, Z.45-8); ibid. I, 78=II, pl. xxx: jw.n $(r)$ djt mtj hıtj NN... $(m)$ jm.s (PI, vso, Z.2-6). Ähnlich ibid. I, $78=$ II, pl. xxix (PI, rto, Z.I6-9). Allerdings ist es in dieser Bedeutung nur in der Fügung $m t j$ hstj 'das Herz zufriedenstellen' belegt, und das trifft auch für das Demotische noch weitgehend zu, vgl. W. Erichsen, Demotisches Glossar (Kopenhagen, 1954), Igo und id. Auswahl frühdemotischer Texte, Heft 2 (Kopenhagen, I950), 62. Es ist eine Wendung, die ganz offensichtlich der Urkundensprache angehört und die deshalb in diesem Kontext gar nicht zu erwarten ist. Außerdem wäre der Sinn der beiden Sätzchen nicht sehr überzeugend.

Es scheint mir einleuchtender, $m t j$ hier als Schreibung von $m t n$, 'belohnen' ( $W b$. II, I7o) aufzufassen:' 'Mögest du belohnen wegen dessen, was ich getan habe, mit einem großen Königtum' bzw., in Z. 38, '〈mit〉 Kraft und Stärke'. Man muß allerdings in diesem Fall vor qn $n h t$ ein $m$ ergänzen. Aber auch Caminos hatte ja ein ganz ähnliches Problem: In einem Fall (Z.35) nimmt er eine nota accusativi $m$ an, im zweiten Fall (Z.38) stünde dagegen ein direktes Objekt. Zudem findet sich in Z.30 eben dieselbe Schreibung qn $n h t$ an einer Stelle, wo unbedingt $\langle m\rangle q n n h t$ zu lesen ist. Es handelt sich also sowohl in Z.35 ( $m$ njswyt) als auch in Z.38 ( $\langle m\rangle q n$ nht) um Präpositionalphrasen, die m.E. als Ergänzung zum Verb $m t(n) . k$ 'belohnen (mit)' gehören.

\section{Eine besondere Schreibung des Infinitivs von jrj, 'machen'}

Im Bildfeld der Stele ist ganz rechts der Hohepriester Jwpwt räuchernd dargestellt; diese

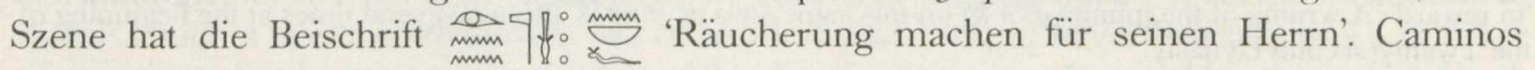
(p. 53) bemerkt sehr treffend: 'The infinitive of iri must be meant, the two $n$ 's after the eye sign being doubtless an error easier to emend than to account for'. Immerhin kann man feststellen, daß diese Schreibung (und vergleichbare wie $\mathrm{mm}$ ) mehrfach belegt ist, und zwar vor allem auf Särgen, die nach den von A. Niwiński aufgestellten Kriterien ${ }^{3}$ in die späte 2I. bis frühe 22. Dynastie gehören, also in etwa dieselbe Zeit wie die Stele:

-2 Belege (jrj.nn qbhw/jrinn sntr) stehen auf einem Sarg im Vatikan, publiziert von J. Capart in Miscellanea Gregoriana (Rom, I94I), 5I-6 (s. Foto auf p. 53).

-3 Belege finden sich auf einem Sarg in Krakau, publiziert von A. Niwiński in BIFAO 86 (I986), 257 ff.; Auf der Ausklapptafel, oben links: jrj.nn sntr; dto., unten rechts: jrj.n sntr; auf pl. xxxviii A: jrj.nn sntr.

- Auf dem Sarg Berlin Nr. 58 (G. Roeder, Aegyptische Inschriften ... Berlin (Leipzig, I924), II, 468, Z.7) ein weiteres Beispiel für jrj.nn sntr.

- 2 unsichere Fälle auf dem Sarg Berlin $\operatorname{IIg78~}_{\text {( } Z \ddot{A} S} 39$ (I9OI), Tafel iv, oben links und Tafel V, oben rechts): jrj.n(n) mnzs (vgl. Wb. II, 88, 8-Io) zu lesen?

- Gleich Io Belege hat der Sarg Nr. 621 des Náprstkovo Museum Prag (M. Verner, Altägyptische Särge in den Museen und Sammlungen der Tschechoslowakei (CAA Prague, I982), 206-80, und swar gibt es hier 7 Schreibungen mit einem $n(\underset{m}{\infty})$ und 3 merkwürdige Kompromißschreibungen mit $n$ und $t(\underset{\sim}{\stackrel{\circ}{m})})$ jij.n sntr: Inschrift $\mathrm{Nr}$. IOO (p. 254); jrj.n(t) sntrr:

\footnotetext{
1 Zum Stamm mtj s. J. Osing, Die Nominalbildung des Ägyptischen (Mainz, I976), 643 ff.

${ }^{2}$ Zum Wegfall des $n$ vgl. Caminos in diesem selben Aufsatz, p. $5^{2}$ oben sowie G. Fecht, Wortakzent und Silbenstruktur (Glückstadt, I960), §162 (mit weiterer Literatur).

${ }^{3} \mathrm{~L} \ddot{A} \mathrm{~V}, 44^{1-4} ; 2$ Ist Dynasty Coffins from Thebes (= Theben 5 ).
} 
Nr. 43 (p. 244); jr. $n m d t$ : Nr. 34 (p. 243), Nr. 59 (p. 246), Nr. 66 (p. 247), Nr. 78 (p. 250), Nr. 84 (p. 251), Nr. 93 (p. 253); jrj.n(t) madt: Nr. 33 und 37 (p. 243).

Es ist nun bemerkenswert, daß einige der Opferdarstellungen dieses Sarges, zu denen die zitierten Infinitive als Beischriften gehören, eben diese Beischriften sogar in doppelter Ausführung tragen, und zwar in unterschiedlicher Orthographie: Parallel zu Nr. 33 (jrj.n(t) $m \underline{d t}$ ) Inschrift Nr. 30 (p. 242 und 263: jrj $m \underline{d t}$, also in der für diese Zeit üblichen Schreibung des Infinitivs ohne $t$ ), parallel zu Nr. $37(j r j . n(t) m d t$ ) Nr. 34 (p. 243 und 263: jrj.n $m \underline{d} t$ ), zu Nr. 66 (jrj.n $m d t$ ) Nr. 62 (p. 247 und 270: jrj $m d t$ ), zu Nr. 84 (jrj.n mdtt) Nr. 85 (p. 25I und 273: $j r j m d t$. Daraus geht deutlich hervor, daß all diese Varianten miteinander austauschbar sind.

Die Schreibung ist aber nicht auf die Übergangszeit 2I./22. Dynastie beschränkt, obwohl sie dort auffallend häufig ist, sie kommt auch später vor:

- Statue Kairo CG 42231 (s. meine Ägyptische Biographien der 22. und 23. Dynastie (Wiesbaden, 1985), 546, oben, Z.4): jrj.n qbhw.

- Block Kopenhagen AEIN I040 (s. L. M. und A. Leahy, $\mathscr{T} E A 72$ (1986), I45): jrj.n htp-dj$n j s w t^{1}$

Die Erklärung all dieser Fälle, die sich zweifellos bei systematischer Suche noch vermehren ließen, kann nur darin liegen, daß wir es hier mit relativ frühen Belegen für den Anschluß des Objekts durch $m / n$ zu tun haben: ${ }^{2}$ Statt des üblichen $j r j$ sntr (z.B.)

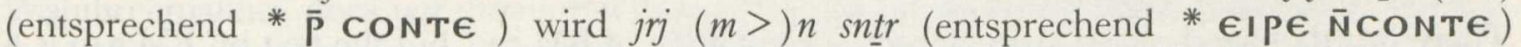
geschrieben. Auffallend ist allerdings, daß diese Schreibung nur mit $j r j$ vorzukommen scheint.

Zweifellos anders gelagerte Fälle sind die Schreibungen des Infinitivs von jrjals der Widmungsformel: jrj.n.f $m$ mnw.f... den Inschriften des Chonstempels am Ende der 20. Dynastie vorkommen, s. The Temple of Khonsu (The Epigraphic Survey), I, pl. 52 (s. dazu p. 28, n.c. im Übersetzungsheft); pl. 53 oben; II, pl. I42, C; pl. I43, A; pl. 195; pl. I96; pl. 202 E. Hier folgt ja jeweils eine mit $n$ beginnende Phrase auf den Infinitiv.

KARL JANSEN-WINKELN 